Journal of Mathematics and Statistics 6 (1): 52-55, 2010

ISSN 1549-3644

(C) 2010 Science Publications

\title{
Exterior Differential Systems for Higher Order Partial Differential Equations
}

\author{
Paul Bracken \\ Department of Mathematics, University of Texas, Edinburg, TX 78541-2999
}

\begin{abstract}
The theory of exterior differential systems is applied to study integrability of a set of related partial differential equations expressed in the terms of differential forms using Cartan's method. The Camassa-Holm equation and the Degasperis-Procesi equations are special cases that are described by these exterior differential systems. Some conservation laws are obtained in the cases of the more relevant equations. A closed differential ideal is constructed for each case studied.
\end{abstract}

Key words: Differential form, partial differential equation, integrability, Camassa-Holm

\section{INTRODUCTION}

It has been known for a while that the simplest nonlinear evolution equations which have solitary wave solutions or solitons are known to have an infinite number of conservation laws. This in turn is related to the concept of integrability of a particular partial differential equation or system of such equations (Estabrook and Wahlquist, 1975). There are many implications and applications of the idea of integrability. For example, a Lax pair can usually be determined and a Backlund or auto-Backlund transformation might be written down.

It was shown by Wahlquist and Estabrook (1975; 1976) that by applying results from the theory of exterior differential systems, as well as prolongation techniques, it is possible to determine integrability of a related partial differential system. The use of prolongation results can often lead to a Lax pair or Backlund transformation for the given nonlinear system.

The equation which was studied by Wahlquist and Estabrook is the classical Korteweg-de Vries equation, which has been known to describe the long time evolution of finite amplitude waves. The equation has many applications which extend beyond the original applications to solitary surface waves. It seems to be that nonlinear dispersion can act to compatify solitary waves and generate solitons with a finite wavelength. Recently a generalized Korteweg-de Vries equation (Rosenau and Hyman, 1993; Sivers and Takens, 1988; Bracken, 2005) has been studied. The symmetry group has been determined for this equation and several classes of solutions have been obtained, which include both soliton solutions and solutions which have compact support (Pikorsky and Rosenau, 2006). The type of approach which was begun by Wahlquist and Estabrook has been applied to this generalized equation as far as determining some prolongations is concerned (Bracken, 2007).

It is the intention here to review some of the mathematical background which will let us study some interrelated equations which have been of interest recently. These ideas are finally applied to a class of equations that includes the Camassa-Holm and Degasperis-Procesi equations. These equations are of the form:

$\left(\mathrm{u}-\mathrm{u}_{\mathrm{xx}}\right)_{\mathrm{t}}+\mathrm{u}\left(\mathrm{u}-\mathrm{u}_{\mathrm{xx}}\right)_{\mathrm{x}}+\beta\left(\mathrm{u}-\mathrm{u}_{\mathrm{xx}}\right) \mathrm{u}_{\mathrm{x}}=0$

for specific choices of the constant $\beta$.

An exterior differential system which reproduces the given equation on the transverse manifold is developed for each case. The derivatives of the forms in this set are shown to be expressible in terms of the same forms, so the integrability of each equation is established. Finally, conservation laws for the two equations will be written down developed from the original set of one-forms.

Differential systems and their equations: Consider the space $M=\mathbb{R}^{n}(x, t, u, p, q, \ldots)$ in which there is defined a closed exterior differential system which will be written:

$\alpha_{1}=0, \ldots, \alpha_{m}=0$

Let $I$ be the ideal generated by the set $\left\{\alpha_{i}\right\}_{i=1}^{m}$ in (2). This is given explicitly as:

$I=\left\{\omega=\sum_{i=1}^{m} \sigma_{i} \wedge \alpha_{i}: \sigma_{i} \in \Lambda(M)\right\}$ 
If the ideal in (3) is closed, it follows that $\mathrm{dI} \subset \mathrm{I}$ and so we may say that (2) is integrable. It is important to stress that system (2) is chosen in such a way that the solutions $\mathrm{u}=\mathrm{u}(\mathrm{x}, \mathrm{t})$ of a specific equation:

$\mathrm{u}_{\mathrm{t}}=\mathrm{F}\left(\mathrm{x}, \mathrm{t}, \mathrm{u}, \mathrm{u}_{\mathrm{x}}, \mathrm{u}_{\mathrm{xx}}, \ldots\right)$

correspond with the two-dimensional integral manifolds of (2). These are the integral manifolds given by sections $\mathrm{S}$ of the projection:

$\pi: \mathrm{M} \rightarrow \mathbb{R}^{2}, \quad \pi(\mathrm{x}, \mathrm{t}, \mathrm{u}, \mathrm{p}, \mathrm{q}, \ldots)=(\mathrm{x}, \mathrm{t})$

These sections $\mathrm{S}$ are given by a mapping:

$\mathrm{S}: \mathbb{R}^{2} \rightarrow \mathrm{M}, \quad \mathrm{S}(\mathrm{x}, \mathrm{t})=(\mathrm{x}, \mathrm{t}, \mathrm{u}(\mathrm{x}, \mathrm{t}), \mathrm{p}(\mathrm{x}, \mathrm{t}), \mathrm{q}(\mathrm{x}, \mathrm{t}), \ldots)$

Due to the required transversality, we have $d x \wedge$ $\left.\mathrm{dt}\right|_{\mathrm{S}}=\pi^{*}(\mathrm{dx} \wedge \mathrm{dt}) \neq 0$.

Several exterior differential systems will be discussed here. It will be seen that we end with a particular differential system which leads to some partial differential equations of interest, in particular, an equation of the form (1):

- Let us introduce the differential system defined to be:

$$
\begin{aligned}
& \alpha_{1}=\mathrm{du} \wedge \mathrm{dt}-\mathrm{pdx} \wedge \mathrm{dt} \\
& \alpha_{2}=\mathrm{dp} \wedge \mathrm{dt}-\mathrm{qdx} \wedge \mathrm{dt} \\
& \alpha_{3}=\mathrm{du} \wedge \mathrm{dx}-\mathrm{dq} \wedge \mathrm{dx}+\mathrm{dq} \wedge \mathrm{dt}+(\mathrm{u}-\mathrm{q}) \mathrm{dx} \wedge \mathrm{dt}
\end{aligned}
$$

The exterior derivatives of the $\beta i$ can be calculated and we obtain:

$$
\begin{aligned}
& \mathrm{d} \alpha_{1}=-\mathrm{dp} \wedge \mathrm{dx} \wedge \mathrm{dt}=\frac{1}{\mathrm{q}} \alpha_{2} \wedge \mathrm{dp} \\
& \mathrm{d} \alpha_{2}=-\mathrm{dq} \wedge \mathrm{dx} \wedge \mathrm{dt}=\alpha_{3} \wedge \mathrm{dx} \\
& \mathrm{d} \alpha_{3}=\mathrm{du} \wedge \mathrm{dx} \wedge \mathrm{dt}-\mathrm{dq} \wedge \mathrm{dx} \wedge \mathrm{dt}=\alpha_{3} \wedge \mathrm{dt}
\end{aligned}
$$

Therefore, it can be seen that all of these exterior derivatives vanish modulo $\left\{\alpha_{j}\right\}_{j=1}^{3}$. Any regular two-dimensional solution manifold in the fivedimensional space $S_{2}=\left\{u(x, t), u_{x}=p(x, t)\right.$, $\left.\mathrm{p}_{\mathrm{x}}(\mathrm{x}, \mathrm{t})=\mathrm{q}(\mathrm{x}, \mathrm{t})\right\}$ satisfying a specific partial differential equation of the form (4) will annul this set of forms. The exact form of this equation which corresponds to (5) can be found explicitly by sectioning the forms into the solution manifold. On
$S_{2}$, we must have $d_{u}=u_{x} d_{x}+u_{t} d t$, and similarly for both $\mathrm{p}$ and $\mathrm{q}$. It follows that:

$$
\begin{aligned}
& 0=\alpha_{1} \mid \mathrm{S}=\left(\mathrm{u}_{\mathrm{x}}-\mathrm{p}\right) \mathrm{dx} \wedge \mathrm{dt} \\
& 0=\alpha_{2} \mid \mathrm{S}=\left(\mathrm{p}_{\mathrm{x}}-\mathrm{q}\right) \mathrm{dx} \wedge \mathrm{dt} \\
& 0=\alpha_{3} \mid \mathrm{S}=\left(\mathrm{u}_{\mathrm{t}}-\mathrm{q}_{\mathrm{t}}-(\mathrm{u}-\mathrm{q})-\mathrm{q}_{\mathrm{x}}\right) \mathrm{dt} \wedge \mathrm{dx}
\end{aligned}
$$

Therefore, $\mathrm{p}=\mathrm{u}_{\mathrm{x}}$ and $\mathrm{q}=\mathrm{u}_{\mathrm{xx}}$ with the equation given by:

$$
\left(\mathrm{u}-\mathrm{u}_{\mathrm{xx}}\right) \mathrm{t}-\left(\mathrm{u}-\mathrm{u}_{\mathrm{xx}}\right)-\mathrm{u}_{\mathrm{xxx}}=0
$$

This is the specific equation whose integrability is implied by system (7)

- Consider the differential system:

$$
\begin{aligned}
& \alpha_{1}=d u \wedge d t-p d x \wedge d t \\
& \alpha_{2}=d p \wedge d t-q d x \wedge d t \\
& \alpha_{3}=d u \wedge d x-d q \wedge d x+d u \wedge d t- \\
& \quad d q \wedge d t+(u-q) d x \wedge d t
\end{aligned}
$$

Differentiating each of these, we obtain:

$$
\begin{aligned}
& \mathrm{d} \alpha_{1}=-\mathrm{dp} \wedge \mathrm{dx} \wedge \mathrm{dt}=\mathrm{dx} \wedge \alpha_{2} \\
& \mathrm{~d} \alpha_{2}=-\mathrm{dq} \wedge \mathrm{dx} \wedge \mathrm{dt}=\mathrm{dx} \wedge\left(-\alpha_{3}+\alpha_{1}\right) \\
& \mathrm{d} \alpha_{3}=-\mathrm{dx} \wedge \alpha_{3}
\end{aligned}
$$

Upon sectioning these forms, we again obtain that $\mathrm{p}=\mathrm{u}_{\mathrm{x}}, \mathrm{q}=\mathrm{u}_{\mathrm{xx}}$ and the equation which pertains to (11) arises from the section $\left.\alpha_{3}\right|_{\mathrm{s}}=0$ and is given by:

$\left(\mathrm{u}-\mathrm{u}_{\mathrm{xx}}\right) \mathrm{t}-\left(\mathrm{u}-\mathrm{u}_{\mathrm{xx}}\right)_{\mathrm{x}}-\left(\mathrm{u}-\mathrm{u}_{\mathrm{xx}}\right)=0$

The final two cases which will be introduced include equations which are being actively studied at the moment

- Let $\beta$ be a real, nonzero constant. Define the exterior differential system as the collection of two-forms

$$
\begin{aligned}
& \alpha_{1}=\mathrm{du} \wedge \mathrm{dt}-\mathrm{pdx} \wedge \mathrm{dt} \\
& \alpha_{2}=\mathrm{dp} \wedge \mathrm{dt}-\mathrm{qdx} \wedge \mathrm{dt} \\
& \alpha_{3}=-\mathrm{du} \wedge \mathrm{dx}+\mathrm{dq} \wedge \mathrm{dx}- \\
& \quad \beta \mathrm{udq} \wedge \mathrm{dt}+\beta(2 \mathrm{u}-\mathrm{q}) \mathrm{du} \wedge \mathrm{dt}
\end{aligned}
$$

The exterior derivatives of the $\alpha_{j}$ are given by: 


$$
\begin{aligned}
\mathrm{d}_{1}= & \mathrm{dx} \wedge \mathrm{dp} \wedge \mathrm{dt}=\mathrm{dx} \wedge \alpha_{2} \\
\mathrm{~d} \alpha_{2}= & \mathrm{dx} \wedge \mathrm{dq} \wedge \mathrm{dt}= \\
& \frac{1}{\beta \mathrm{u}} \mathrm{dx} \wedge\left(-\alpha_{3}+\beta \mathrm{u} \alpha_{1}+\beta(\mathrm{u}-\mathrm{q}) \alpha_{1}\right) \\
\mathrm{d} \alpha_{3}= & 0
\end{aligned}
$$

These vanish modulo the set of $\alpha_{j}$ from (16) and carrying out the sectioning, the equation is obtained from the restriction of $\alpha_{3}$ :

$$
\left.\alpha_{3}\right|_{s}=\left(u_{t}-q_{t}+\beta u_{x}-\beta u_{x}+\beta(u-q) u_{x}\right) d x \wedge d t
$$

Using $\mathrm{p}=\mathrm{u}_{\mathrm{x}}$ and $\mathrm{q}=\mathrm{u}_{\mathrm{xx}}$ from sectioning $\alpha_{1}$ and $\alpha_{2}$, we have finally:

$\left(\mathrm{u}-\mathrm{u}_{\mathrm{xx}}\right) \mathrm{t}+\beta\left(\mathrm{u}\left(\mathrm{u}-\mathrm{u}_{\mathrm{xx}}\right)\right)_{\mathrm{x}}=0$

- The following system leads to an important class of partial differential equations which are of much current interest. The Camassa-Holm and Degasperis-Procesi equations are two particular examples which appear. Define the system of forms:

$$
\begin{aligned}
\alpha_{1}= & d u \wedge d t-p d x \wedge d t \\
\alpha_{2}= & d p \wedge d t-q d x \wedge d t \\
\alpha_{3}= & -d u \wedge d x+d q \wedge d x-u d q \wedge d t+ \\
& \quad u d u \wedge d t+\beta(u-q) d u \wedge d t
\end{aligned}
$$

The quantity $\beta$ which appears in $\alpha_{3}$ is a real, nonzero constant. Differentiating each of these, we have:

$$
\begin{aligned}
\mathrm{d} \alpha_{1}= & -\mathrm{dp} \wedge \mathrm{dx} \wedge \mathrm{dt}=\mathrm{dx} \wedge \alpha_{2} \\
\mathrm{~d} \alpha_{2}= & 1 \mathrm{dx} \wedge\left(-\alpha_{3}+\mathrm{u}((1+\beta) \mathrm{u}-\mathrm{q}) \alpha_{1}\right) \\
\mathrm{d} \alpha_{3}= & (1-\beta) \mathrm{dq} \wedge \mathrm{du} \wedge \mathrm{dt} \\
= & (1-\beta) \mathrm{dq} \wedge \alpha_{1}+(1-\beta) \mathrm{pdq} \wedge \mathrm{dx} \wedge \mathrm{dt} \\
= & (1-\beta) \mathrm{dq} \wedge \alpha_{1}+(1-\beta) \mathrm{p}\left(\alpha_{3}+\mathrm{du} \wedge \mathrm{dx}\right) \wedge \mathrm{dt} \\
= & (1-\beta) \mathrm{dq} \wedge \alpha_{1}+(1-\beta) \mathrm{pdt} \wedge \alpha_{3} \\
& -(1-\beta) \mathrm{d} \mathrm{dx} \wedge \mathrm{du} \wedge \mathrm{dt} \\
= & (1-\beta)\left[\mathrm{dq} \wedge \alpha_{1}+\mathrm{pdt} \wedge \alpha_{3}-\mathrm{pdx} \wedge \alpha_{1}\right]
\end{aligned}
$$

All of the details for calculating $d \alpha_{3}$ have been shown here. Clearly all of the $d \alpha_{j}$ vanish modulo the set of $\alpha_{j}$. Sectioning $\alpha_{1}$ and $\alpha_{2}$ gives $p=u_{x}$ and $q=u_{x x}$ as in the other cases and the equation results from evaluating the section as follows:

$$
0=\left.\alpha_{3}\right|_{\mathrm{s}}=\left(\mathrm{u}_{\mathrm{t}}-\mathrm{q}_{\mathrm{t}}+\mathrm{u}\left(\mathrm{u}_{\mathrm{x}}-\mathrm{q}_{\mathrm{x}}\right)+\beta(\mathrm{u}-\mathrm{q}) \mathrm{u}_{\mathrm{x}}\right) \mathrm{dx} \wedge \mathrm{dt}
$$

These results imply the partial differential equation:

$(\mathrm{u}-\mathrm{q})_{\mathrm{t}}+\mathrm{u}(\mathrm{u}-\mathrm{q})_{\mathrm{x}}+\beta(\mathrm{u}-\mathrm{q}) \mathrm{u}_{\mathrm{x}}=0$

Substituting $\mathrm{q}=\mathrm{u}_{\mathrm{xx}}$ into (19), an equation of the form (1) is obtained:

$\left(u-u_{x x}\right) t+u\left(u-u_{x x}\right)_{x}+\beta\left(u-u_{x x}\right) u_{x}=0$

If we introduce $\rho=u-u_{x x}$, then for the case $\beta=3$, Eq. 20 becomes the Degasperis-Procesi equation:

$\rho_{\mathrm{t}}+\rho_{\mathrm{x}} \mathrm{u}+3 \rho \mathrm{u}_{\mathrm{x}}=0, \rho=\mathrm{u}-\mathrm{u}_{\mathrm{xx}}$

Finally, for $\beta=2$, (20) is the Camassa-Holm equation:

$$
\rho_{\mathrm{t}}+\rho_{\mathrm{x}} \mathrm{u}+2 \rho \mathrm{u}_{\mathrm{x}}=0, \rho=\mathrm{u}-\mathrm{u}_{\mathrm{xx}}
$$

Conservation laws: Consider now the related subject of conservation laws for these systems. Conservation laws associated with the equations of the last two cases correspond to the existence of exact two-forms contained in the ring of the forms $\left\{\alpha_{j}\right\}$. Let us suppose that we can find a set of functions $f_{i}(x, t, u, p, q)$ such that the two-form:

$\sigma=\mathrm{f}_{1} \alpha_{1}+\mathrm{f}_{2} \alpha_{2}+\mathrm{f}_{3} \alpha_{3}$

satisfies $d \sigma=0$, the condition for exactness. This is the integrability condition for the existence of a one-form $\omega$ such that:

$\sigma=\mathrm{d} \omega$

which conversely implies that $\mathrm{d} \sigma=0$. Only the final two equations which were discussed in Section 2 will be considered now.

For case (iii), consider the one-form $\sigma$ given by:

$\sigma=\alpha_{3}$

It has already been shown by differentiating $\sigma$ that $\mathrm{d} \sigma=0$. In fact, $\beta$ can be derived from a single oneform. Let $\omega$ be defined to be:

$\omega=(q-u) d x+\beta u(u-q) d t$ obtain:

By working out the exterior derivative of $\omega$, we 


$$
\begin{aligned}
\mathrm{d} \omega= & -\mathrm{du} \wedge \mathrm{dx}+\mathrm{dq} \wedge \mathrm{dx}+\beta \mathrm{udu} \wedge \mathrm{dt}-\beta \mathrm{udq} \wedge \mathrm{dt}+ \\
& \beta(\mathrm{u}-\mathrm{q}) \mathrm{du} \wedge \mathrm{dt}
\end{aligned}
$$

This is exactly the form $\sigma$ in (25).

With regard to case (iv), with the $\left\{\alpha_{i}\right\}$ given by (17), we can similarly define a form $\sigma$. To do calculations here, the exterior derivatives of the forms in (17) can be simplified to read:

$$
\begin{aligned}
\mathrm{d} \alpha_{1} & =-\mathrm{dp} \wedge \mathrm{dx} \wedge \mathrm{dt}, \mathrm{d} \alpha_{2}=-\mathrm{dq} \wedge \mathrm{dx} \wedge \mathrm{dt}, \mathrm{d} \alpha_{3} \\
& =(1-\beta) \mathrm{dq} \wedge \mathrm{du} \wedge \mathrm{dt}
\end{aligned}
$$

In this case, consider the one-form $\beta$ which is defined to be:

$\sigma=\alpha_{3}-(1-\beta) q \alpha_{1}+(1-\beta) p \alpha_{2}$

Calculating the exterior derivatives of $\sigma$, it is found that it vanishes:

$$
\begin{aligned}
\mathrm{d} \sigma= & (1-\beta) \mathrm{dq} \wedge \mathrm{du} \wedge \mathrm{dt}-(1-\beta) \mathrm{dq} \wedge \mathrm{du} \wedge \mathrm{dt}+ \\
& (1-\beta) \mathrm{ddq} \wedge \mathrm{dx} \wedge \mathrm{dt}+(1-\beta) \mathrm{qdp} \wedge \mathrm{dx} \wedge \mathrm{dt}- \\
& (1-\beta) \mathrm{qdp} \wedge \mathrm{dx} \wedge \mathrm{dt}-(1-\beta) \mathrm{pdq} \wedge \mathrm{dx} \wedge \mathrm{dt}=0
\end{aligned}
$$

Now it can be shown that $\beta$ can be derived from a one-form, namely $\omega$ defined by:

$$
\omega=(q-u) d x+\frac{1}{2}\left(u^{2}-2 u q+\beta u^{2}+p^{2}\right) d t
$$

Differentiating $\omega$, it is obtained that:

$$
\begin{gathered}
\mathrm{d} \omega=-\mathrm{du} \wedge \mathrm{dx}+\mathrm{dq} \wedge \mathrm{dx}+\mathrm{udu} \wedge \mathrm{dt}-\mathrm{udq} \wedge \mathrm{dt}+ \\
\quad \beta \mathrm{udu} \wedge \mathrm{dt}-\mathrm{qdu} \wedge \mathrm{dt}+(1-\beta) \mathrm{pdp} \wedge \mathrm{dt}
\end{gathered}
$$

This is precisely the two-form $\sigma$ given in (27).

The associated conservation law results from an application of Stokes theorem, which is:

$$
\oint_{\mathrm{M}_{1}} \omega=\oint_{\mathrm{M}_{2}} \mathrm{~d} \omega
$$

This has been written for any simply-connected, two-dimensional manifold $\mathrm{M}_{2}$ with closed onedimensional boundary $\mathrm{M}_{1}$. The notation implies that $\omega$ and $\mathrm{d} \omega$ are to be evaluated on their respective manifolds.

Considering $\omega$ again, we can of course add to this $\omega$ any exact one-form $\mathrm{dv}$, where $\mathrm{v}$ is an arbitrary, scalar function. Thus $\omega$ can be taken to be: $\omega=d v+(q-u) d x+\frac{1}{2}\left(u^{2}-2 u q+\beta u^{2}+p^{2}\right) d t$

such that $\sigma=\mathrm{d} \omega$. Now $v$ may be regarded simply as a coordinate in an extended six-dimensional space of variables $\{\mathrm{x}, \mathrm{t}, \mathrm{u}, \mathrm{p}, \mathrm{q}, \mathrm{v}\}$ and the one-form $\omega$ may be included with the original set of forms. Since $d \omega$ is known to be in the ring of the original set, the new set of forms remains a closed ideal.

\section{REFERENCES}

Bracken, P., 2005. Symmetry properties of a generalized Korteweg-de Vries equation and some explicit solutions. Int. J. Math. Math. Sci., 13: 2159-2173.

Bracken, P., 2007. An exterior differential system for a generalized Korteweg-de Vries equation and its associated integrability. Acta Applied Math., 95: 223-231.

Estabrook, F.B. and H.D. Wahlquist, 1975. The geometric approach to sets of ordinary differential equations and Hamiltonian dynamics. SIAM Rev., 17: 201-220.

Pikorsky, A. and P. Rosenau, 2006. Phase compactons. Phys. D., 218: 56-69.

Rosenau, P. and J. Hyman, 1993. Compactons: Solitons with finite wavelength. Phys. Rev. Lett., 70: 564-567.

Sivers, A.J. and S. Takens, 1988. Intrinsic localized modes in anharmonic crystals. Phys. Rev. Lett., 61: 970-973.

Wahlquist, H.D. and F.B. Estabrook, 1975. Proplongation structures of nonlinear evolution equations. J. Math. Phys., 16: 1-7.

Wahlquist, H.D. and F.B. Estabrook, 1976. Prolongation structures of nonlinear evolution equations II. J. Math. Phys., 17: 1293-1297. 